\title{
ORIGINAL ARTICLE \\ Genetic screening reveals a link between Wnt signaling and antitubulin drugs
}

\author{
$\mathrm{AH} \mathrm{Khan}^{1}$, JS Bloom ${ }^{2}$, E Faridmoayer ${ }^{1}$ and DJ Smith ${ }^{1}$
}

The antitubulin drugs, paclitaxel (PX) and colchicine (COL), inhibit cell growth and are therapeutically valuable. PX stabilizes microtubules, while $\mathrm{COL}$ promotes their depolymerization. But, the drug concentrations that alter tubulin polymerization are hundreds of times higher than their clinically useful levels. To map genetic targets for drug action at single-gene resolution, we used a human radiation hybrid panel. We identified loci that affected cell survival in the presence of five compounds of medical relevance. For PX and COL, the zinc and ring finger 3 (ZNRF3) gene dominated the genetic landscape at therapeutic concentrations. ZNRF3 encodes an R-spondin regulated receptor that inhibits Wingless/Int (Wnt) signaling. Overexpression of the ZNRF3 gene shielded cells from antitubulin drug action, while small interfering RNA knockdowns resulted in sensitization. Further a potent pharmacological inhibitor of Wnt signaling, Wnt-C59, protected cells from PX and COL. Our results suggest that the antitubulin drugs perturb microtubule dynamics, thereby influencing Wnt signaling.

The Pharmacogenomics Journal (2016) 16, 164-172; doi:10.1038/tpj.2015.50; published online 7 July 2015

\section{INTRODUCTION}

Although the human genome was declared fully sequenced in $2003,{ }^{1}$ we still know little of the role of individual genes in key aspects of mammalian cell biology and physiology. This gap in our knowledge is of particular concern in the area of drug design, particularly in identifying specific genetic targets for medicinal compounds.

For decades the standard approaches for identifying mammalian drug targets have required assays tailor-made for each compound. These techniques include ligand binding, cross-linking and mass spectrometry. ${ }^{2,3}$ A more versatile strategy to identify drug targets can be found in genetics. Genome-wide association studies have identified loci for drug-related phenotypes in humans, mice and lympoblastoid cells. ${ }^{4-11}$ However, the yield of genetic loci has been low. In addition, successes have generally been limited to the idiosyncratic side effects of drugs. The modest return of genetics in understanding drug action may reflect the intense evolutionary pressure on natural polymorphisms, which limits genetic variation. Moreover, naturally occurring polymorphisms do not produce equivalent functional alterations across the genome.

'Knockdowns' using interfering RNA can test genes in a relatively unbiased fashion. ${ }^{12,13}$ However, these screens use separate interfering RNA molecules to study each of the 20000 mammalian genes and are hence expensive, laborious and time consuming. Recently, scientists have used CRISPR/Cas9 editing technologies to find genes for drug action. ${ }^{14-16}$ These experiments have been fruitful, but rely on complex lentiviral libraries of unknown stability. Also, each gene knockout makes up only a small proportion of the cell pool under drug selection. The limited representation can cause bias. Further, screening approaches are limited to known genes.
Radiation hybrid $(\mathrm{RH})$ mapping was originally invented as a technique to determine the relative locations of genes within mammalian genomes ${ }^{17}$ and became an essential tool in the sequencing of the human genome. ${ }^{18} \mathrm{RH}$ panels are constructed by lethally irradiating cells, shattering the DNA. The DNA fragments are then introduced into living cells so that a random assortment of genes are triploid rather than diploid. ${ }^{19,20}$ (For the sex chromosomes, the additional copies are diploid rather than haploid.) Each $\mathrm{RH}$ clone contains $\sim 10-30 \%$ of the genome as extra copies, with the whole genome represented many times in the entire panel of $\sim 100$ clones. Significantly, the triploid segments are small $(\sim 10 \mathrm{Mb})$, exhibiting a large number of breakpoints $\left(\sim 10^{4}\right)$. This property affords the technique very high mapping accuracy. In fact, $\mathrm{RH}$ mapping can localize markers to within a single gene. Further, the broad sampling of the genome in a high proportion of cells lends robustness to the $\mathrm{RH}$ approach.

After the genome was sequenced, RH libraries were no longer needed for gene mapping. Moreover, although $\mathrm{RH}$ panels were used in the sequencing process, only the DNA of cells was scrutinized; other gene-linked characteristics of the cell were not investigated widely. Functional assays in $\mathrm{RH}$ panels have been used in a few studies to identify genes for metabolic disorders $^{21}$ and viral receptors. ${ }^{22,23}$ Our group has also employed $\mathrm{RH}$ panels to dissect transcriptional regulatory mechanisms and mammalian genetic interactions. ${ }^{19,20,24,25}$ The $\mathrm{RH}$ approach can thus offer the benefits of an unbiased dissection of gene/ phenotype relationships, without the need to rely on idiosyncratic natural polymorphisms or incomplete gene annotations. In this paper, we employ RH panels as a cost-effective and efficient tool to identify mammalian drug targets at single-gene resolution.



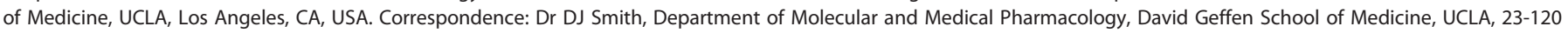
CHS, Box 951735, Los Angeles, CA 90095-1735, USA. 


\section{MATERIALS AND METHODS}

Cells

$\mathrm{RH}$ clones from the human/hamster G3 pane $^{26}$ were propagated in a minimum essential medium with $10 \%$ fetal bovine serum, $1 \times$ penicillin/ streptomycin (100 units $\mathrm{ml}^{-1}$ penicillin, $100 \mathrm{\mu g} \mathrm{ml}^{-1}$ streptomycin; Life Technologies, Grand Island, NY, USA) and $1 \times$ hypoxanthine-aminopterinthymidine (HAT; $100 \mu \mathrm{m}$ hypoxanthine, $0.4 \mu \mathrm{m}$ aminopterin and $16 \mu \mathrm{m}$ thymidine; Life Technologies). ${ }^{19,20} \mathrm{~A}$ total of 79 clones was available. Before doing the drug assays, cells were weaned from HAT by growing in the medium for 1 week, then for 2 weeks in HT medium lacking aminopterin, and then for 1 week in nonsupplemented medium. ${ }^{27}$ The A23 hamster cells are the recipient cells used to construct $\mathrm{RH}$ panels and were grown in non-HAT medium. HEK293 cells were grown in Dulbecco's modified Eagle's medium with $10 \%$ fetal bovine serum and $1 \times$ penicillin/streptomycin.

The G3 RH panel was obtained from Dr A. Dusty Miller, Fred Hutchinson Cancer Research Center, Seattle, WA. The A23 cells were obtained from Dr Christine Farr, University of Cambridge, Cambridge, UK. The HEK293 cells were obtained from the American Type Culture Collection, Manassas, VA.

At the conclusion of the experiments, the identities of the HEK293 and A23 cells were verified by simple tandem repeat genotyping (IDEXX BioResearch, Columbia, MO, USA). The HEK293 cells were identical with the reported genetic profile of this line, with the exception of one missing allele each at markers CSF1PO and D5S818. There was no mammalian interspecies contamination. The A23 cells were confirmed to be of Chinese hamster origin with no mammalian interspecies contamination. Cells were not tested for mycoplasma contamination.

\section{Drug assays in $\mathrm{RH}$ cells}

Methotrexate (MTX), 6-mercaptopurine, paclitaxel (PX; Taxol) and colchicine (COL) were obtained from Tocris Bioscience (Bristol, UK). Dimethyl sulfoxide (DMSO) was from Thermo Scientific (Waltham, MA, USA).

We performed drug assays in 96-well plates by seeding $10^{4} \mathrm{RH}$ cells into each well. To minimize variation, individual $\mathrm{RH}$ clones were distributed into wells from the same cell suspension. We allowed cells to grow for $4 \mathrm{~h}$ before adding drugs. To aid solubilization, drugs were dissolved in DMSO and then diluted in medium. The final concentration of DMSO in all assays was $0.37 \%$, except for the experiments where the DMSO concentration was allowed to vary. We allowed cells to grow in the presence of drug for $24 \mathrm{~h}$, before measuring proliferation using CellTiter-Glo (Promega, Madison, WI, USA) following the manufacturer's instructions. To correct for the different growth rates of the $\mathrm{RH}$ clones and for batch effects due to pipetting, results from individual clones were normalized to vehicle-treated cells of the same clone and batch.

Pilot experiments were used to identify an optimum range of concentrations for each drug ( $n=3$ per concentration) using clone $\mathrm{RH} 23$. To screen the RH panel, we used 2-6 replicates per cell line at each concentration (Supplementary Information).

\section{Mapping}

We previously determined genotypes for 235790 markers in the G3 RH panel using array comparative genomic hybridization (aCGH). ${ }^{19}$ We binned the copy number of each marker into 0 or 1 extra copies (diploid or triploid) for mapping, and used coordinates from the GRCh37/hg19 genome assembly (https://genome.ucsc.edu).

We discarded triploid markers present in either less than five clones or in all clones. We also discarded markers with identical genotypes. A total of 54826 markers remained. Logarithm of the odds scores were computed as described. ${ }^{28,29}$ Genome-wide significance levels were set by permuting the phenotype data 1000 times for each scan. The permutation procedure maintained the correlation structure of the phenotypes, but kept the genotypes unaltered. We used logarithm of the odds scores corresponding to a 5 or $10 \%$ family-wise error rate (FWER) as thresholds for genome-wide significant and suggestive loci, respectively. Computational analyses used $R^{30}$

We calculated statistical power by spiking in a locus of defined narrowsense heritability $\left(h^{2}\right)$ at random locations in the genome. A total of 100 simulations was performed for each value of $h^{2}$ and significance thresholds obtained by 500 permutations of the phenotype data.

\section{Heritability}

Broad-sense heritabilities $\left(H^{2}\right)$ were computed using standard approaches that compare the genetic component of variance among different clones to the total variance. ${ }^{28,31}$
ZNRF3 and DHFR copy number

We used our published aCGH data ${ }^{19}$ to evaluate the continuous genotypes of the RH clones classified as either harboring two or three copies of the ZNRF3 gene (Ensembl: ENSG00000183579, Entrez Gene: 84133) or the dihydrofolate reductase (DHFR) gene (Ensembl: ENSG00000228716, Entrez Gene: 1719). The number of clones classified as having an extra copy of ZNRF3 was nine and the number having an extra copy of DHFR was seven.

Half maximal effective concentration ( $\mathrm{EC}_{50}$ )

The $\mathrm{EC}_{50}$ for each of the compounds was calculated using the drFitSpline function of the grofit package in $\mathrm{R}^{32}$

\section{ZNRF3 expression in $\mathrm{RH}$ cells}

We quantitated expression of the ZNRF3 gene in RH cells using TaqMan (Life Technologies, Grand Island, NY, USA) real-time quantitative PCR (qPCR). Cells were grown in $75-\mathrm{cm}^{2}$ tissue culture flasks using medium containing $25 \mathrm{~nm} \mathrm{PX}, 25 \mathrm{~nm}$ COL or vehicle (0.37\% DMSO). To control for the amount of RNA in each qPCR reaction, we measured expression of the housekeeping gene glyceraldehyde 3-phosphate dehydrogenase (GAPDH; Ensembl: ENSG00000111640, Entrez Gene: 2597). Primer sets were employed in which the amplification and probe oligonucleotides recognized sequences fully conserved between the human and hamster ZNRF3 and GAPDH genes.

ZNRF3 oligos were sense amplification, 5'-TCCTTGTCAAAATCAAGCTGAA GC-3'; antisense amplification, 5'-TGCTCTTGGAGTTGAACTTTCTG-3'; probe, 5'-ATTCCATGAACAGGCTGGCTGTGCAGG-3'. GAPDH oligos were sense amplification, 5'-TCTTCCAGGAGCGAGATCCC-3'; antisense amplification, 5'-AACATGGGGGCATCAGCAG-3'; probe, 5'-CACCATGGAGAAGGCTGGGGC-3'. To avert detection of contaminating genomic DNA, the amplification primers spanned at least one exon junction. Each qPCR reaction used $100 \mathrm{ng}$ of total RNA.

The 9 clones in the panel with an extra copy of ZNRF3 were used for analysis. In addition, 14 clones without an extra copy of ZNRF3 were randomly chosen using the sample function of the base package in $R$. There was non-planned exclusion of one clone with an extra copy of ZNRF3 from the PX category and two clones with an extra copy of ZNRF3 from the COL category, because of outlier values ( $>7$ s.d.).

\section{ZNRF3 verification}

We overexpressed the ZNRF3 gene using a TrueORF Gold CDNA clone (catalog number RC219546, OriGene Technologies, Rockville, MD, USA). The clone is expression validated and sequence verified. The ZNRF3 CDNA in this plasmid is driven by the cytomegalovirus promoter. Knockdowns of the ZNRF3 gene used the small interfering RNA (siRNA) ZNRF3-2 (sense, 5'-GCUGCUACACUGAGGACUATT-3'; antisense, 5'-UAGUCCUCAGUGUAGC AGCCG-3'; Sigma, Life Science, St Louis, MO, USA). ${ }^{33}$

For either CDNA overexpression or siRNA knockdowns, we seeded $10^{4}$ HEK293 or A23 cells into 96-well plates. After $24 \mathrm{~h}, 50 \mathrm{ng}$ of ZNRF3 CDNA clone was transfected into each well using the Lipofectamine RNAiMAX kit (Life Technologies, Grand Island, NY, USA), following the manufacturer's instructions. For the siRNA experiments, we transfected 1 pm of siRNA into each well using the same kit. Antitubulin drugs were added at the same time as the transfection reagents. We evaluated cell proliferation $24 \mathrm{~h}$ later using CellTiter-Glo ( $n=6$ for experiment and controls and each drug and cell line). Cell growth was normalized to vehicle-treated cells in the same batch of experiments. In the overexpression experiments, mock transfection employed pCMV-GFP (plasmid 11153, Addgene, Cambridge, MA, USA), a plasmid in which the green fluorescent protein is driven by the cytomegalovirus promoter. In the knockdown experiments, mock transfection used scrambled siRNA (sense, 5'-CACUUCACGGAUAGGAAUUUGCTT-3'; antisense, 5'-GCAAAUUCCUAUCAUCCGUGAAGUGTT-3').

We evaluated overexpression and knockdown of ZNRF3 in HEK293 cells using TaqMan qPCR ( $n=3$ for experiment and controls). The primer set Hs00393094_m1 was used to measure ZNRF3 mRNA (Life Technologies). We measured expression of GAPDH using primer set Hs99999905_m1. The primer sets for ZNRF3 and GAPDH spanned an exon junction, preventing amplification of contaminating genomic DNA.

We added Wnt-C59 to cells simultaneously with the antitubulin drugs. We used the same protocol as the transfection experiments, but the transfection reagent and nucleic acids were omitted ( $n=3$ for experiment and controls). 


\section{Statistics}

Quoted sample sizes represent biological replicates. Significance thresholds for the quantitative trait locus (QTL) mapping employed permutation. Other evaluations of statistical significance used two-tailed $t$-tests or analysis of variance, as appropriate, employing the $t$-test or aov functions, respectively, in the stats package in $R$.

Values from qPCR as well as our published microarray and aCGH data sets ${ }^{19}$ were log transformed before statistical testing to improve normality and homogeneity of variances. Normality was evaluated with the ShapiroWilk test employing shapiro.test of the stats package in R. ${ }^{30,34}$ Inhomogeneity of the variances was evaluated with Levene's test employing leveneTest of the car package in $\mathrm{R}^{35}$ Despite the transformation, the microarray and $\mathrm{aCGH}$ data showed strong departures from normality (expression levels of DHFR, $W=0.6904, P=1.3 \times 10^{-11}$ ) and homogeneity of variances (continuous copy number values, $F_{[1,77]}=26.0, P=2.43 \times 10^{-6}$ for ZNRF3; $F_{[1,77]}=102.1, P=9.05 \times 10^{-16}$ for $\left.D H F R\right)$. We therefore employed the Kruskal-Wallis test to analyze these data, using the function kruskal.test of the stats package in R.

Observations were plotted as means \pm s.e.m. The QTL analysis used permutation, a nonparametric approach to testing statistical significance. Statistical power for the RH panel also employed permutation. In addition, a post hoc test was performed to evaluate the power to detect expression differences of ZNRF3 in the RH panel. We used pwr.t2n.test of the pwr package in . $^{36}$ Formal power analysis was not undertaken for the other experiments, but sample sizes were based on similar studies in the literature.

Code and data availability

Computer code in $\mathrm{R}$ and experimental data are available in the Supplementary Information.

\section{RESULTS}

\section{Statistical power}

To map QTLs for cell growth in the presence of drugs, we used the G3 human/hamster RH panel. ${ }^{26}$ The G3 panel has 79 clones and was previously genotyped by our group using $(\mathrm{aCGH}) .{ }^{19}$ We classified nearly 55000 markers in the panel as being either triploid or diploid. On average, each clone contained $11 \%$ of the genome as triploid,

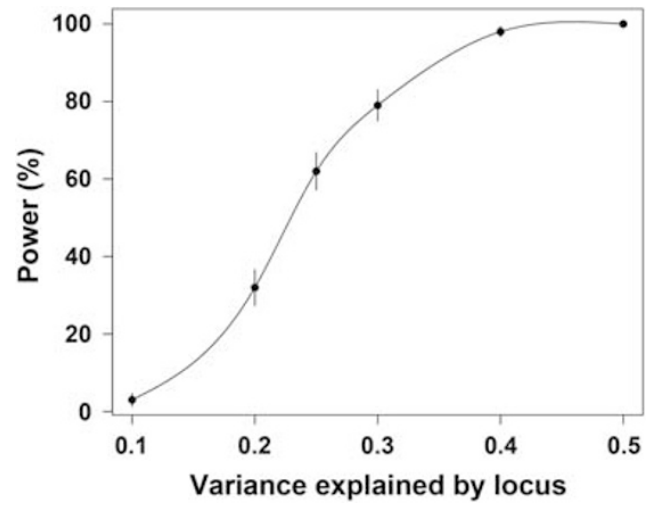

Figure 1. Statistical power; means \pm s.e.m. and the human genome is thus represented $9 \times$ in the entire panel. The average length of the triploid segments was $4 \mathrm{Mb}$.

We estimated statistical power to map a randomly placed single QTL at various narrow-sense heritabilities. ${ }^{28,29}$ Permutation was used to set genome-wide significance thresholds at a FWER of $<5 \%$. The $\mathrm{RH}$ panel had $32 \%$ power to detect a locus that contributed $20 \%$ to the variance and $79 \%$ power to detect a locus that contributed $30 \%$ (Figure 1). At a lower heritability of $10 \%$, more typical of a complex trait, ${ }^{28,37}$ the power was only $3 \%$. The limited power of the G3 panel reflected the relatively small number of clones, but suggested that the panel may be able to detect individual loci of strong effect size.

\section{Drugs}

To identify drug targets, we exposed the G3 panel to antiproliferative drugs. A wide variety of these important agents are available and are used to treat diseases ranging from cancer to autoimmunity. We examined five compounds-MTX, 6-mercaptopurine, PX (Taxol), COL and DMSO. ${ }^{4,5,8,9,38}$

MTX acts by inhibiting DHFR, which is necessary for de novo synthesis of thymidine and purines. Similarly, the agent 6mercaptopurine and its metabolites inhibit the de novo pathway for purine ribonucleotide synthesis.

PX and COL are antitubulin drugs, but have opposite modes of action. PX stabilizes microtubules, while COL induces their depolymerization. We reasoned that these antitubulin agents may perturb common pathways, despite their differing mechanisms.

DMSO is a solvent that has been used to facilitate transdermal delivery of therapeutic agents. The compound also has antiinflammatory, antioxidant and antiproliferative properties. ${ }^{39}$ As well as identifying DMSO target genes, we examined this agent to ensure that QTLs found with the other compounds were not due to DMSO in the vehicle.

The $\mathrm{RH}$ clones had been grown in HAT medium. Aminopterin inhibits DHFR, the same target as MTX. We therefore weaned cells from the HAT medium before performing the drug assays.

We performed pilot experiments to identify a range of concentrations for each drug that spanned the lowest and highest growth rates. Serial dilution was used to create a range of 12 concentrations for each compound. We then tested the series on an arbitrarily chosen clone, $\mathrm{RH} 23$. After $24 \mathrm{~h}$ in the drugs, cell growth was measured using CellTiter-Glo. This reagent evaluates the number of metabolically active cells based on luminescent measurement of ATP. We corrected for differing RH clone growth rates by normalizing to cells treated with vehicle from the same clone and batch.

To screen the entire $\mathrm{RH}$ panel, we chose seven drug concentrations that spanned the $\mathrm{EC}_{50}$ of each drug. The concentrations of compounds used in the screen are shown in Table 1. Survival curves for clone $\mathrm{RH} 23$ are shown in Figure 2a.

Correlations between drugs

To look for similarities in drug action, we examined correlations in cell growth across the $\mathrm{RH}$ panel for each pair of compounds.

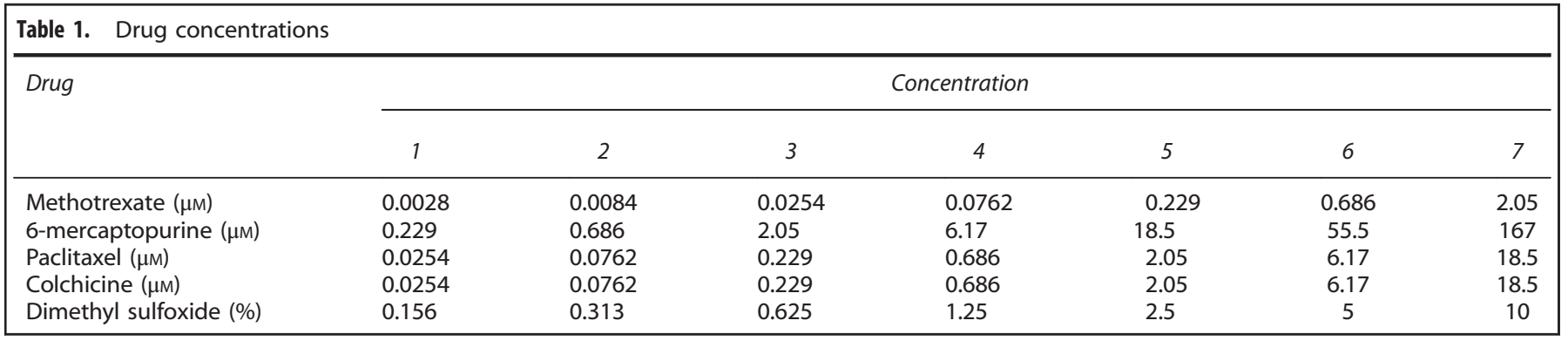



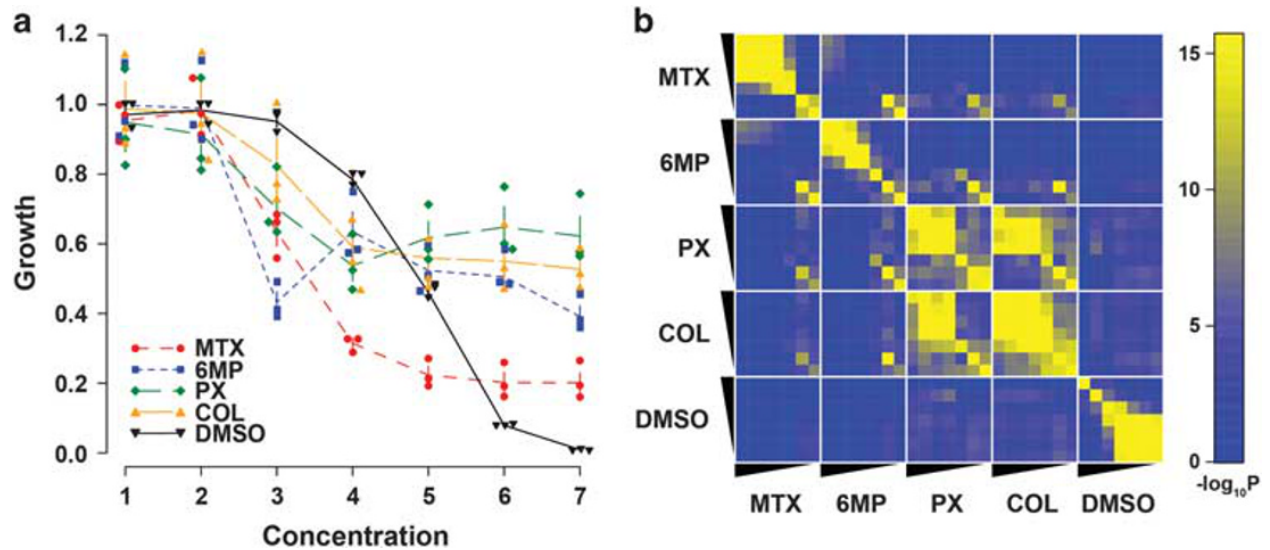

Figure 2. Survival curves and drug correlations. (a) Survival curves for RH23; means \pm s.e.m. (b) $P$-values for drug correlations across RH panel on a negative logarithmic scale. The thin ends of the wedges correspond to lowest drug concentrations. $6 \mathrm{MP}$, 6-mercaptopurine; COL, colchicine; DMSO, dimethyl sulfoxide; MTX, methotrexate; PX, paclitaxel; $\mathrm{RH}$, radiation hybrid.


Figure 3. Growth variations and broad-sense heritabilities. (a) Normalized growth variation among RH clones in paclitaxel, $25 \mathrm{~nm}$; means \pm s.e.m. (b) Normalized growth variation among RH clones in colchicine, $25 \mathrm{~nm}$; means \pm s.e.m. (c) Broad-sense heritabilties, $H^{2}$, at various drug concentrations; means \pm s.e.m. $\mathrm{RH}$, radiation hybrid.

From Figure $2 b$, it is clear that the effects of $\mathrm{PX}$ and $\mathrm{COL}$ are most alike (comparing $\mathrm{PX}$ and $\mathrm{COL}$, mean $R=0.63 \pm 0.02$ s.e.m.; comparing all other drugs, mean $R=0.27 \pm 8.8 \times 10^{-3}$ s.e.m.; Welch's two-tailed $t$-test, $\left.t_{[1,65.6]}=15.5, P<2.2 \times 10^{-16}\right)$. Both PX and $\mathrm{COL}$ act on microtubules, even though with differing mechanisms.

\section{Broad-sense heritabilities}

We found significant variation among the growth rates of the $\mathrm{RH}$ clones in the compounds. Examples for $\mathrm{PX} \quad\left(F_{[78,224]}=2.41\right.$, $\left.P=2.27 \times 10^{-7}\right)$ and $\operatorname{COL}\left(F_{[78,224]}=2.78, P=1.83 \times 10^{-9}\right)$ at $25 \mathrm{~nm}$ are shown in Figures $3 a$ and $b$. We calculated the broad-sense heritabilities for each of the compounds at the seven concentrations (Figure 3c). As drug concentrations increased, broad-sense heritabilities were overall roughly stable $\left(F_{[6,70]}=0.85, P=0.53\right)$. The mean $\mathrm{H}^{2}$ was $33 \% \pm 2.6 \%$ s.e.m. (range $27-41 \%$ ) at the lowest concentration, and $55 \% \pm 4.7 \%$ s.e.m. (range 39-68\%) at the highest. However, PX and COL showed significant increases in broad-sense heritabilities as their concentrations increased $\left(F_{[6,14]}=9.1, P=3.6 \times 10^{-4}, \mathrm{PX} ; F_{[6,14]}=4.8, P=7.6 \times 10^{-3}, \mathrm{COL}\right)$.

\section{Drug QTLs}

We mapped QTLs for cell growth in the five agents at the seven concentrations. We also mapped QTLs for $\mathrm{EC}_{50}$. To get FWERs, we used a permutation procedure similar to that employed for the power analysis. We found five suggestive QTLs, with a genomewide significance of $<10 \%$ (Table 2). Of these, two loci passed the accepted threshold for genome-wide significance of $<5 \%$.

PX and COL share a QTL in the ZNRF3 gene

At therapeutic levels of $25 \mathrm{~nm}, \mathrm{PX}$ and COL each had a QTL significant at $<5 \%$ on chromosome 22 (Figures $4 a$ and $b$ ). The 1logarithm of the odds support interval of the two QTLs was $\sim 50$ $\mathrm{kb}{ }^{40}$ This resolution was sufficient to localize both loci to a single gene, ZNRF3 (Figure 4c). The ZNRF3 gene product is a potent inhibitor of Wnt signaling. ${ }^{33,41}$ The shared QTL of the two drugs suggests that they act by a common mechanism that perturbs Wnt pathways at clinically relevant concentrations.

An extra copy of ZNRF3 increased cell survival in both PX $\left(t_{[1,77]}=5.38, P=7.8 \times 10^{-7}\right)$ and $\operatorname{COL}\left(t_{[1,77]}=5.36, P=8.5 \times 10^{-7}\right.$; Figures $5 \mathrm{a}$ and b). The narrow-sense heritability $\left(h^{2}\right)$ of this locus contributed $27 \%( \pm 0.9 \%$ s.e.m. $)$ to the variance of both drugs. Further, the narrow-sense heritabilies of ZNRF3 were similar to the broad-sense heritabilities $\left(H^{2}\right)$ of the drugs (PX $H^{2}=27 \% \pm 3.1 \%$ s.e.m., $\mathrm{COL} H^{2}=32 \% \pm 3.3 \%$ s.e.m.). Because of the Beavis effect (the so-called 'winner's curse'), the narrow-sense heritability of a first-discovered locus is frequently overestimated. ${ }^{42}$ Nevertheless, ZNRF3 dominates the genetic landscape at therapeutic concentrations of PX and COL, and this locus may account for a substantial part of the overall genetic variance. 
Table 2. Significant and suggestive loci

\begin{tabular}{lllllll}
\hline Chr & Peak marker (chr coord, bp) & Compound & Conc & LOD & FWER (\%) & Gene \\
\hline 3 & 2142282 & Paclitaxel & $76 \mathrm{~nm}$ & 5.2 & $<10$ & CNTN4 \\
4 & 107670990 & Methotrexate & $0.7 \mu \mathrm{m}$ & 4.8 & $<10$ & Chr4_54.10 (hypothetical) \\
11 & 27182677 & DMSO & $10 \%$ & 5.3 & $<10$ & Chrr11.469 (hypothetical) \\
22 & 29315203 & Paclitaxel & $25 \mathrm{~nm}$ & 5.5 & $<1$ & ZNRF3 \\
22 & 29342619 & Colchicine & $25 \mathrm{nM}$ & 5.4 & $<5$ & ZNRF3 \\
\hline
\end{tabular}

Abbreviations: Chr, chromosome; chr coord, chromosome coordinate; Conc, concentration; DMSO, dimethyl sulfoxide; FWER, family-wise error rate; LOD, logarithm of the odds.

a



b

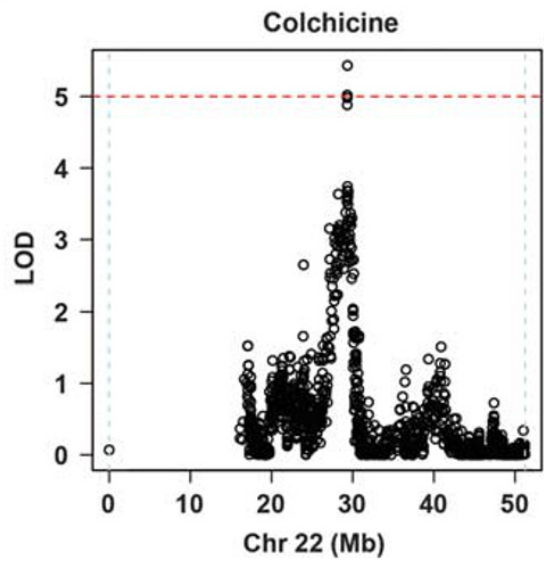

C

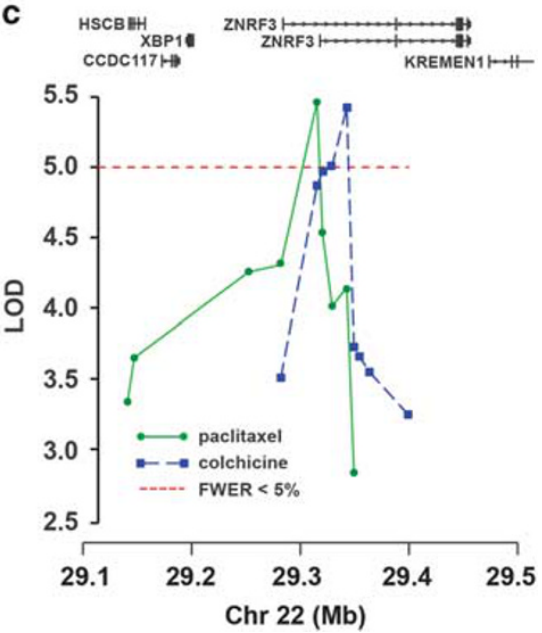

Figure 4. Chromosome 22 locus for paclitaxel and colchicine. (a) Paclitaxel, 25 nm. (b) Colchicine, 25 nm. (c) Closeup of locus. University of California, Santa Cruz (UCSC) genes at top. Red horizontal lines, FWER $<5 \%$ threshold. FWER, family-wise error rate.
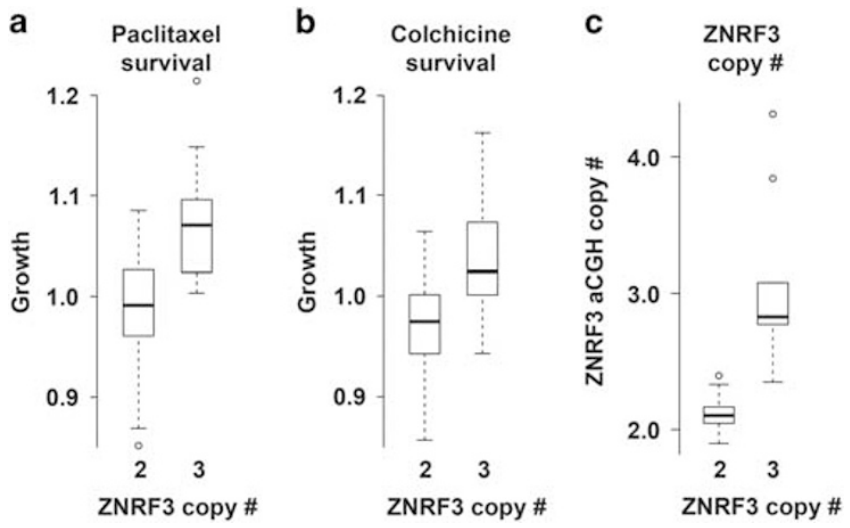

Figure 5. ZNRF3. (a) Effect of ZNRF3 on cell survival in paclitaxel. Proliferation relative to vehicle-treated control. (b) Effect of ZNRF3 on cell survival in colchicine. (c) ZNRF3 copy number. Comparing classified copy number with array comparative genomic hybridization $(\mathrm{aCGH})$ copy number.

We compared the genotypes of the $\mathrm{RH}$ clones classified as either harboring two or three copies of the ZNRF3 gene with the continuous copy number data from aCGH. ${ }^{19}$ The classified data was consistent with the continuous data (Figure 5c). There was a mean copy number of $2.1 \pm 0.01$ s.e.m. for clones classified as harboring two copies of ZNRF3 and $3.0 \pm 0.22$ s.e.m. for clones classified as harboring three (KruskalWallis test, $X_{[\mathrm{d} f=1, N=79]}^{2}=23.2, P=1.5 \times 10^{-6}$ ).
There was no information on ZNRF3 in our pre-existing expression data for the $\mathrm{RH}$ panel, as the microarray we used did not contain the gene. ${ }^{19}$ We therefore employed real-time qPCR to examine ZNRF3 expression in the RH panel. We evaluated clones with or without an extra copy of ZNRF3 in medium supplemented with $25 \mathrm{~nm}$ PX, $25 \mathrm{~nm}$ COL or vehicle. Surprisingly, we found no effect of an extra gene copy or drug status on ZNRF3 expression $\left(F_{[1,21]}=1.40, P=0.25\right.$ for $Z N R F 3$ status in vehicle; $F_{[1,20]}=0.29$, $P=0.60$ for $Z N R F 3$ status in $P X ; F_{[1,19]}=2.38, P=0.14$ for $Z N R F 3$ status in $C O L ; F_{[1,63]}=2.25,0.89$ and $P=0.14,0.35$ for main effects of $\mathrm{PX}$ and $\mathrm{COL}$, respectively; all planned comparisons; Supplementary Figure S2).

A caveat to our conclusions is the limited statistical power of the experiment. A post hoc analysis gave a two-tailed power to detect a main effect of ZNRF3 on expression as $20.2 \%$. Nevertheless, the observations raise the possibility that the extra copy of ZNRF3 exerts its effects at the protein level, perhaps as a result of altered translational efficiency or due to amino acid differences between human and hamster.

\section{Suggestive QTLS}

We found three other QTLs that exceeded a suggestive genomewide significance threshold of $<10 \%$ (Table 2). There was a locus for PX at $76 \mathrm{~nm}$ on chromosome 3 (Figure 6a). The peak marker was in the contactin 4 (CNTN4) gene. CNTN4 belongs to the immunoglobulin superfamily and encodes an axon-associated cell adhesion molecule. Haploinsufficiency of CNTN4 has been implicated in autism spectrum disorders. ${ }^{43,44}$ 
a

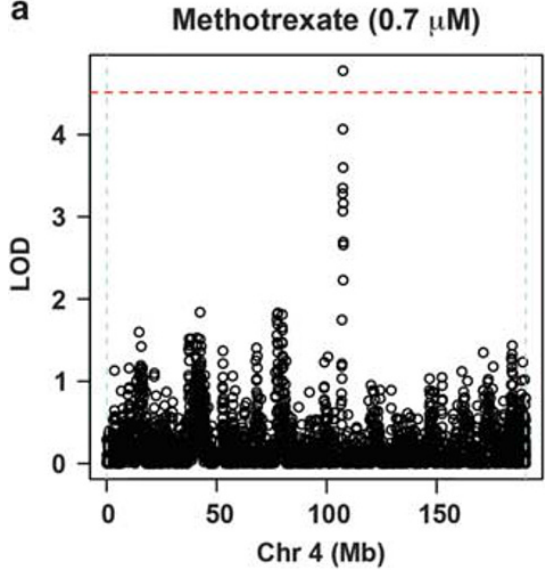

b



C

DMSO (10\%)

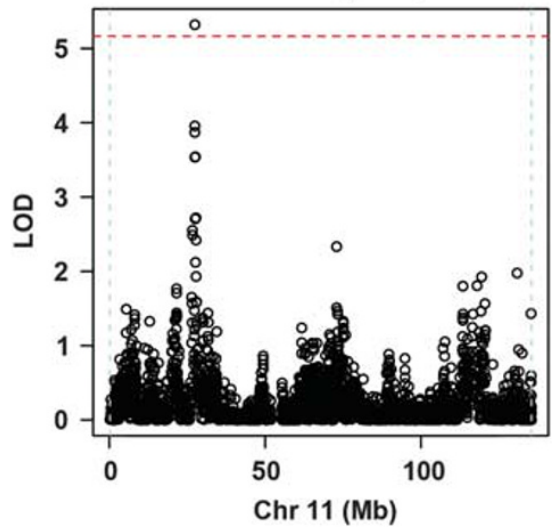

Figure 6. Suggestive QTLs. (a) Paclitaxel, $76 \mathrm{~nm}$. (b) Methotrexate, $0.7 \mu \mathrm{m}$. (c) DMSO, 10\%. Red horizontal lines, FWER < 10\% threshold. DMSO, dimethyl sulfoxide; FWER, family-wise error rate; QTL, quantitative trait locus.

Suggestive loci for MTX at $0.7 \mu \mathrm{M}$ and DMSO at $10 \%$ were found on chromosomes 4 and 11 , respectively (Figures $6 \mathrm{~b}$ and c). In both cases, the peak marker resided in a hypothetical gene. There were no QTLs for DMSO that overlapped with loci for the other compounds, verifying that vehicle artifacts were not an issue in our study.

There was no QTL for MTX at its target gene, DHFR. The RH panel was originally propagated in HAT medium, which includes the drug aminopterin. Similar to MTX, aminopterin inhibits DHFR and cells can respond to long-term culture in either drug by multicopy amplification of the DHFR gene. ${ }^{45-47}$ We reasoned that culture of the RH panel in HAT medium may have amplified the DHFR gene, preventing detection of a MTX QTL.

To test this conjecture, we examined our published aCGH and transcript-profiling data from the $\mathrm{RH}$ panel. ${ }^{19}$ There was no amplification of the DHFR gene. The genotypes of the $\mathrm{RH}$ clones classified as either harboring two or three copies of the DHFR gene were congruent with the continuous copy number estimates from aCGH. In fact, the mean copy number from aCGH was $2.0 \pm 0.008$ s.e.m. for clones classified as having two copies of $D H F R$, and $2.9 \pm 0.22$ s.e.m. for clones classified as having three (Kruskal-Wallis test, $X_{[\mathrm{d} f=1, N=79]}^{2}=18.9, P=1.4 \times 10^{-5}$; Supplementary Figure S1A).

Consistent with the lack of evidence for DHFR gene amplification, there was no significant difference in the expression of the gene between clones with two copies (relative expression = $1.2 \pm 0.16$ s.e.m.) and three (expression $=1.1 \pm 0.28$ s.e.m.; KruskalWallis test, $X_{[\mathrm{d} f=1, N=79]}^{2}=0, P=1$; Supplementary Figure $\mathrm{S} 1 \mathrm{~B}$ ).

We also looked for QTLs that regulated the $\mathrm{EC}_{50}$ of the five compounds. We found no loci that exceeded the suggestive genome-wide FWER of $<10 \%$.

Validating ZNRF3 in the action of the antitubulin drugs

To confirm the role of ZNRF3 in the effects of PX and COL, we overexpressed the gene in tissue culture cells using a cytomegalovirus promoter. Real-time qPCR was used to verify overexpression in transfected HEK293 cells compared with mock-transfected cells (11.3-fold \pm 2.8 s.e.m., Student's two-tailed $t$-test, $t_{[1,4]}=8.44$, $P=0.0011$; Figure 7a). Overexpression of ZNRF3 protected HEK293 (Figure 7b) and A23 cells (Figure 7c) from the actions of PX $\quad\left(F_{[1,50]}=18.7, P=7.3 \times 10^{-5}\right.$, HEK293 cells; $F_{[1,50]}=23.5$, $P=1.3 \times 10^{-5}$, A23 cells $)$ and COL $\left(F_{[1,50]}=4.7, P=0.034\right.$, HEK293 cells; $F_{[1,50]}=9.1, P=4.0 \times 10^{-3}, A 23$ cells). In fact, the effect of ZNRF3 overexpression on growth of HEK293 cells in $25 \mathrm{~nm}$ PX (1.11-fold enhanced growth \pm 0.05 s.e.m.) and in $25 \mathrm{~nm}$ COL (1.05-fold enhanced growth \pm 0.04 s.e.m.) and on growth of A23 cells in $25 \mathrm{~nm}$ PX (1.13-fold enhanced growth \pm 0.06 s.e.m.) and in $25 \mathrm{~nm} \mathrm{COL} \mathrm{(1.09-fold} \mathrm{enhanced} \mathrm{growth} \pm 0.04$ s.e.m.) was similar to the effect of an extra copy of ZNRF3 in the RH panel (1.08-fold enhanced growth \pm 0.02 s.e.m., both drugs).

Conversely, diminished ZNRF3 expression should sensitize cells to growth inhibition by antitubulin drugs. Real-time PCR confirmed decreased expression of ZNRF3 in siRNA-treated HEK293 cells compared with mock-transfected cells $(62 \% \pm 8 \%$ s.e.m., Student's two-tailed $t$-test, $t_{[1,4]}=3.26, \quad P=0.031 i^{33}$ Figure 7a). As expected, ZNRF3 knockdown sensitized HEK293 cells to the actions of PX $\left(F_{[1,50]}=9.9, P=2.9 \times 10^{-3}\right)$ and COL $\left(F_{[1,50]}=18.6, P=7.6 \times 10^{-5} ;\right.$ Figure $\left.7 d\right)$.

Overexpression of ZNRF3 inhibits Wnt signaling. We therefore supposed that pharmacological blockade of the Wnt pathway should protect cells from the antitubulin drugs. To test this idea, we treated cells with the compound Wnt-C59, a potent inhibitor of Wnt signaling. Wnt-C59 blocks porcupine, an enzyme necessary for palmitoylation and biological activity of Wnt. ${ }^{48}$ As expected, Wnt-C59 alone inhibited the growth of HEK293 cells $\left(F_{[1,7]}=10.3\right.$, $P=0.015$; Supplementary Figure S3). However, when further combined with PX or COL, Wnt-C59 diminished the antiproliferative effects of the antitubulin drugs $\left(F_{[2,30]}=43.5, P=1.4 \times 10^{-9}\right.$, $\mathrm{PX} ; F_{[2,30]}=16.8, P=1.3 \times 10^{-5}, \mathrm{COL} ;$ Figure $\left.7 \mathrm{e}\right)$.

\section{DISCUSSION}

We used a human RH panel to identify loci influencing cell survival in the presence of antiproliferative drugs. As we performed a total of 40 assays (five compounds $\times$ eight conditions, consisting of seven concentrations plus the $\mathrm{EC}_{50}$ ), we would expect a total of four false-positive results at a FWER of $<10 \%$. In fact, we identified five suggestive QTLs. The limited yield of the investigation reflected its modest statistical power. However, we were also motivated by the high resolution of $\mathrm{RH}$ panels, even with a small number of clones. Our reasoning was vindicated by the singlegene accuracy of our results.

Despite the restricted power of the study, the antitubulin drugs PX and COL each shared a QTL with a genome-wide significance of $<5 \%$. The QTLs were located in the ZNRF3 gene, an inhibitor of Wnt signaling. The fact that these agents shared the same QTL, bolsters our confidence in ZNRF3 being an important target for the antitubulin drugs. We further verified the role of ZNRF3 by overexpression and knockdown experiments. The compound Wnt-C59 offered additional evidence for the importance of Wnt signaling in antitubulin drug action. 




b
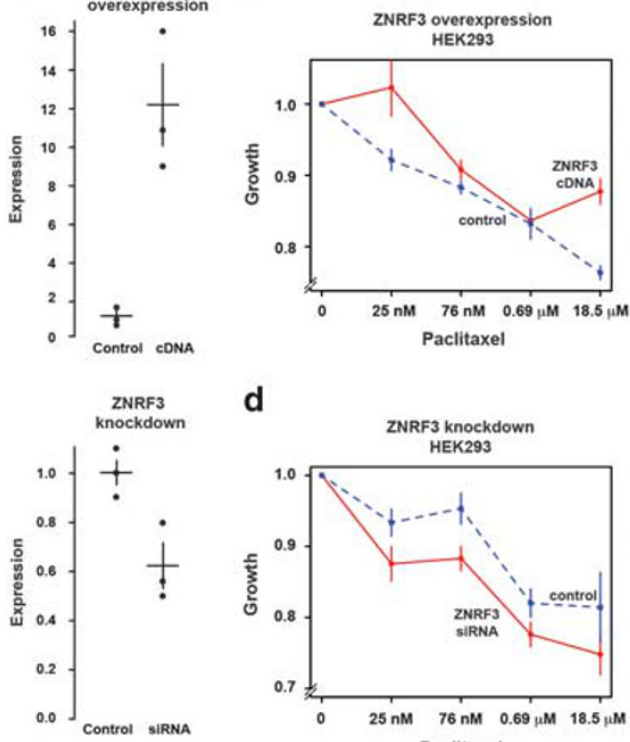

d







C
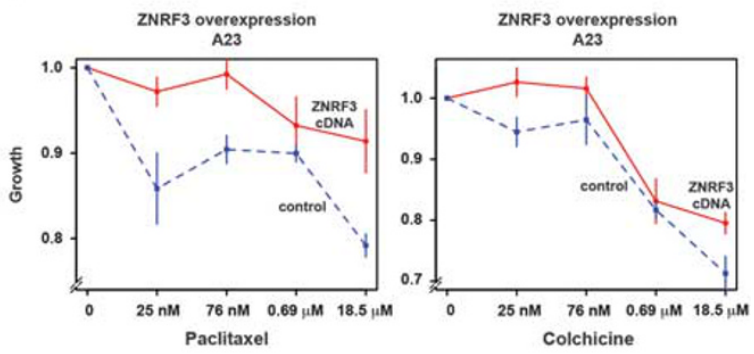

e



Figure 7. Validating ZNRF3 in the action of the antitubulin drugs. (a) Verification of ZNRF3 overexpression and small interfering RNA (siRNA) knockdown in HEK293 cells using quantitative PCR. (b) ZNRF3 overexpression diminishes the effects of the antitubulin drugs in HEK293 cells. (c) ZNRF3 overexpression also decreases the effects of the drugs in A23 cells. (d) Knockdown of ZNRF3 expression with an siRNA sensitizes HEK293 cells to paclitaxel and colchicine. (e) Pharmacological blockade of Wnt signaling with Wnt-C59 protects HEK293 cells from the actions of paclitaxel and colchicine. Growth curves normalized to zero drug concentrations; means \pm s.e.m.

Both PX and COL inhibit microtubules at micromolar concentration. Paradoxically, however, the therapeutic effects of these drugs are obtained at concentrations 10- to a 100-fold lower (tens of nanomolar). ${ }^{13,49-55}$ In fact, when used at concentrations that inhibit microtubules, the drugs become toxic. ${ }^{56,57}$ Antitubulin drugs likely act by altering microtubule dynamics rather than affecting structural integrity. ${ }^{58}$

The fact that we did not identify other components of the Wnt signaling pathway in our screen is consistent with the idea that ZNRF3 is the major locus regulating cell proliferation in PX and $\mathrm{COL}$ at therapeutic concentrations. The dominant role of ZNRF3 is implied by our finding that the narrow-sense heritability of the gene accounts for nearly the entire broad-sense heritability of growth in the presence of the drugs. The dramatic effect of WntC59 on the antitubulin drug response presumably reflects its highaffinity blockade of Wnt pathways, overwhelming the effects of the endogenous genes.

Although ZNRF3 dominated the genetic landscape at therapeutic concentrations, the QTL was not significant at higher concentrations. The disappearance of the QTL was not due to decreased heritability. On the contrary, the broad-sense heritabilities of PX and COL climbed as drug concentrations increased. Perhaps, the antitubulin drugs recruit greater numbers of interacting genes at higher concentrations, resulting in enhanced genetic complexity and decreased power.

The ZNRF3 protein contains an extracellular domain, one transmembrane region and a cytoplasmic RING domain. 33,41,59,60 ZNRF3 negatively regulates Wnt signaling by acting as an E3 ubiquitin ligase that ubiquitinates the frizzled (FZD) family of Wnt receptors. The FZD protein is thus consigned to the lysosome for destruction. R-spondin is a growth factor that inhibits ZNRF3 action by binding to its extracellular domain, thereby activating Wnt signaling. ZNRF3 is frequently mutated in adrenocortical carcinoma, consistent with a role as a tumor-suppressor gene. ${ }^{59,61}$ The inhibitory effects of ZNRF3 on Wnt signaling has lead to suggestions that, when intact, ZNRF3 may be an attractive target for cancer therapy. ${ }^{33}$
Wnt signaling pathways are traditionally classified as either canonical or noncanonical. The noncanonical pathways further belong either to the Wnt/planar cell polarity (Wnt/PCP) pathway or the Wnt/calcium (Wnt/Ca ${ }^{2+}$ ) pathway. The canonical Wnt pathway directly regulates gene transcription, the Wnt/PCP pathway affects the cytoskeleton and the $\mathrm{Wnt} / \mathrm{Ca}^{2+}$ pathway regulates intracellular calcium. In addition, the canonical Wnt pathway exerts its effects through $\beta$-catenin, while the noncanonical pathways do not.

The canonical and noncanonical pathways are initiated by binding of Wnt to FZD. ${ }^{62-65}$ By inhibiting FZD expression, ZNRF3 regulates both the canonical and Wnt/PCP pathways ${ }^{33}$ and is expected to regulate the $\mathrm{Wnt} / \mathrm{Ca}^{2+}$ pathway by the same mechanism. Humans possess a total of $19 \mathrm{Wnt}$ and 10 FZD family members. Membrane bound co-receptors for Wnt, such as lipoprotein receptor-related proteins 5 and 6 (LRP)-5/6, receptor tyrosine kinase and ROR2, associate with FZD to promote Wnt signaling.

In the canonical pathway, absence of Wnt causes phosphorylation, ubiquitination and destruction of $\beta$-catenin by the proteasome. The phosphorylation is the task of the destruction complex, which includes Axin-1, adenomatosis polyposis coli, protein phosphatase 2 A, glycogen synthase kinase 3 (GSK3) and casein kinase $1{ }^{66}$ When Wnt binds to FZD and its co-receptors LRP-5/6, the intracellular domain of LRP6 becomes phosphorylated by GSK3 and casein kinase 1. The receptor complex then internalizes to form a signalosome, consisting of FZD, phospho-LRP6, Dishevelled, GSK3 and Axin-1. Phospho-LRP6 directly inhibits GSK3, thereby inhibiting the phosphorylation and degradation of $\beta$-catenin. The $\beta$-catenin can now localize to the nucleus, where it regulates gene expression by forming complexes with the T-cell factor/lymphoid-enhancing factor family of transcription factors.

The noncanonical pathways are activated when a subset of WNTs, including WNT5A and WNT11, bind FZD. For the Wnt/ PCP pathway, the relevant co-receptors are receptor tyrosine kinase and ROR2 instead of LRP-5/6. Upon engagement of Wnt with its receptors, Dishevelled binds the dishevelledassociated activator of morphogenesis 1. Dishevelled-associated activator of morphogenesis 1 in turn activates the small G-proteins 
RhoA and Rac1, as well as JNK and additional proteins involved in cytoskeletal rearrangement. In contrast, the $\mathrm{Wnt} / \mathrm{Ca}^{2+}$ signaling pathway activates phospholipase $C$, which catalyzes formation of inositol 1,4,5-triphosphate and 1,2 diacylglycerol from membranebound phospholipid phosphatidylinositol 4,5-bisphosphate. The elevated inositol 1,4,5-triphosphate increases intracellular $\mathrm{Ca}^{2+}$ by release from stores in the endoplasmic reticulum. As a result, calmodulin-dependent protein kinase II, protein kinase $C$, the nuclear factor of activated T-cell transcription factor, as well as other transcription factors, are activated.

Our identification of ZNRF3 as the principal QTL at therapeutic concentrations suggests that antitubulin drugs ultimately exert their effects through Wnt signaling. Lending credence to this notion, the canonical Wnt pathway inhibits GSK3, which results in decreased phosphorylation of microtubule-associated protein 1B and stabilization of microtubules. ${ }^{62,67-70}$ In addition, Wnt signaling proteins such as Dishevelled and $\beta$-catenin appear to bind components of the microtubule apparatus. ${ }^{65}$ Cilia prominently feature microtubules and can strongly regulate Wnt pathways. ${ }^{71-75}$ We suggest that these organelles may mediate the action of antitubulin drugs.

Activation of $\beta$-catenin by the canonical Wnt pathway causes increased transcription of c-Myc, which in turn promotes expression of cyclin D1, a key driver of the G1/S transition. ${ }^{65}$ Inhibiting Wnt action thus represses the cell cycle. Consistent with this observation, we found decreased proliferation of cells treated with Wnt-C59. As Wnt-C59 and the antitubulin drugs each repress cell growth, combining the two drug classes might naively be expected to result in additive inhibition of proliferation. In contrast, our ZNRF3 overexpression and siRNA experiments suggested that combining Wnt-C59 and the microtubule-binding agents should block the antiproliferative effects of PX and COL. Our results clearly supported the second alternative and were consistent with the idea that the antitubulin drugs act on Wnt signaling pathways.

PX and COL are the prototypical antitubulin drugs. These agents were hence attractive candidates for initial study using the $\mathrm{RH}$ approach. However, there are a wide variety $(>40)$ of other antitubulin agents. ${ }^{58}$ The vinca alkaloids promote depolymerization similar to $\mathrm{COL}$, but bind to a different site on the microtubule. In addition, compounds such as laulimalide, peloruside A, estramustine and noscapine bind to sites on tubulin distinct from $\mathrm{PX}, \mathrm{COL}$ or the vinca alkaloids. All these compounds may repay study using $\mathrm{RH}$ cells.

The antitubulin drugs are widely employed in the clinic. PX is used in cancer and COL in rheumatology. Our findings therefore encourage the pursuit of drugs that perturb Wnt pathways. ${ }^{62,76}$ Our results also suggest that pharmacological antagonists of Wnt signaling may be useful in treating overdoses of the antitubulin drugs. 77,78

A number of genes relevant to PX action have been identified by siRNA screens. ${ }^{13,55}$ One of the screens, ${ }^{13}$ included siRNAs for ZNRF3, but did not flag the gene as a hit. Perhaps the chosen siRNAs were ineffective at knocking down ZNRF3.

$\mathrm{RH}$ panels show considerable long-range linkage disequilibrium. ${ }^{25}$ This phenomenon appeared unlikely to be a cause of false-positive QTLs in our study. We performed a simulation using a locus spiked into random locations in the genome with a narrow-sense heritability of the same size as ZNRF3 $\left(h^{2}=0.27\right)$. When the genuine QTL was insignificant, genome-wide significant 'ghost' QTLs occurred elsewhere in the genome at a rate of $4 \% \pm 2 \%$ s.e.m. However, even such apparently spurious QTLs may be in the same pathway as the genuine locus. ${ }^{25}$

Together our results suggest that $\mathrm{RH}$ panels can be an efficient method to identify QTLs for drug action. RH panels have the advantages of high resolution, modest labor costs to screen a small panel and an unbiased search of the whole genome. Using a human $\mathrm{RH}$ panel, we were able to identify an important gene for the action of antitubulin drugs that had been overlooked by other strategies. The limited statistical power of existing $\mathrm{RH}$ panels can be overcome in the future by using large pools of $\mathrm{RH}$ clones in bulk segregant analysis. ${ }^{79,80}$ These strategies have very high resolution and statistical power, and will provide a 'one flask' approach to mapping genes for mammalian drug action.

\section{CONFLICT OF INTEREST}

The authors declare no conflict of interest.

\section{ACKNOWLEDGMENTS}

We thank Amela Karagic, Nate Nowak and Sara Zabihi for tissue culture work, Nisar Farhat for coding, and Jake Lusis and Richard Wang for helpful suggestions. This study was supported in part by the National Institutes of Health (R01 GM098273 and R21 HG007405 to DJS)

\section{REFERENCES}

1 Collins FS, Green ED, Guttmacher AE, Guyer MS, US National Human Genome Research Institute. A vision for the future of genomics research. Nature 2003; 422 : 835-847.

2 Fang Y. Ligand-receptor interaction platforms and their applications for drug discovery. Expert Opin Drug Discov 2012; 7: 969-988.

3 Pacholarz KJ, Garlish RA, Taylor RJ, Barran PE. Mass spectrometry based tools to investigate protein-ligand interactions for drug discovery. Chem Soc Rev 2012; 41: 4335-4355.

4 Choy E, Yelensky R, Bonakdar S, Plenge RM, Saxena R, De Jager PL et al. Genetic analysis of human traits in vitro: drug response and gene expression in lymphoblastoid cell lines. PLoS Genet 2008; 4: e1000287.

5 Duan S, Bleibel WK, Huang RS, Shukla SJ, Wu X, Badner JA et al. Mapping genes that contribute to daunorubicin-induced cytotoxicity. Cancer Res 2007; 67: 5425-5433.

6 Eadon MT, Wheeler HE, Stark AL, Zhang X, Moen EL, Delaney SM et al. Genetic and epigenetic variants contributing to clofarabine cytotoxicity. Hum Mol Genet 2013; 22: 4007-4020.

7 Harrill AH, Watkins PB, Su S, Ross PK, Harbourt DE, Stylianou IM et al. Mouse population-guided resequencing reveals that variants in cd44 contribute to acetaminophen-induced liver injury in humans. Genome Res 2009; 19: 1507-1515.

8 Huang RS, Duan S, Bleibel WK, Kistner EO, Zhang W, Clark TA et al. A genomewide approach to identify genetic variants that contribute to etoposide-induced cytotoxicity. Proc Natl Acad Sci USA 2007; 104: 9758-9763.

9 Huang RS, Ratain MJ. Pharmacogenetics and pharmacogenomics of anticancer agents. CA Cancer J Clin 2009; 59: 42-55.

$10 \mathrm{Ni}$ X, Zhang W, Huang RS. Pharmacogenomics discovery and implementation in genome-wide association studies era. Wiley Interdiscip Rev Syst Biol Med 2013; 5: 1-9.

11 Stark AL, Dolan ME. Lymphoblastoid cell lines in pharmacogenomics: how applicable are they to clinical outcomes? Pharmacogenomics 2013; 14: 447-450.

12 Sliva K, Schnierle BS. Selective gene silencing by viral delivery of short hairpin RNA. Virol J 2010; 7: 248.

13 Whitehurst AW, Bodemann BO, Cardenas J, Ferguson D, Girard L, Peyton M et al. Synthetic lethal screen identification of chemosensitizer loci in cancer cells. Nature 2007; 446: 815-819.

14 Wang T, Wei JJ, Sabatini DM, Lander ES. Genetic screens in human cells using the CRISPR-Cas9 system. Science 2014; 343: 80-84.

15 Shalem O, Sanjana NE, Hartenian E, Shi X, Scott DA, Mikkelsen TS et al. Genomescale CRISPR-Cas9 knockout screening in human cells. Science 2014; 343: 84-87.

16 Koike-Yusa H, Li Y, Tan EP, Velasco-Herrera MDC, Yusa K. Genome-wide recessive genetic screening in mammalian cells with a lentiviral CRISPR-guide RNA library. Nat Biotechnol 2014; 32: 267-273.

17 Goss SJ, Harris H. New method for mapping genes in human chromosomes. Nature 1975; 255: 680-684.

18 Cox DR, Green ED, Lander ES, Cohen D, Myers RM. Assessing mapping progress in the human genome project. Science 1994; 265: 2031-2032.

19 Wang RT, Ahn S, Park CC, Khan AH, Lange K, Smith DJ. Effects of genome-wide copy number variation on expression in mammalian cells. BMC Genomics 2011; 12: 562.

20 Park CC, Ahn S, Bloom JS, Lin A, Wang RT, Wu T et al. Fine mapping of regulatory loci for mammalian gene expression using radiation hybrids. Nat Genet 2008; 40: 421-429.

21 De Lonlay P, Mugnier C, Sanlaville D, Chantrel-Groussard K, Bénit P, Lebon S et al. Cell complementation using Genebridge 4 human:rodent hybrids for physical mapping of novel mitochondrial respiratory chain deficiency genes. Hum Mol Genet 2002; 11: 3273-3281. 
22 Ross SR, Schofield JJ, Farr CJ, Bucan M. Mouse transferrin receptor 1 is the cell entry receptor for mouse mammary tumor virus. Proc Natl Acad Sci USA 2002; 99: 12386-12390.

23 Rasko JE, Battini JL, Kruglyak L, Cox DR, Miller AD. Precise gene localization by phenotypic assay of radiation hybrid cells. Proc Natl Acad Sci USA 2000; 97: 7388-7392.

24 Ahn S, Wang RT, Park CC, Lin A, Leahy RM, Lange K et al. Directed mammalian gene regulatory networks using expression and comparative genomic hybridization microarray data from radiation hybrids. PLoS Comput Biol 2009; 5: e1000407.

25 Lin A, Wang RT, Ahn S, Park CC, Smith DJ. A genome-wide map of human genetic interactions inferred from radiation hybrid genotypes. Genome Res 2010; 20: 1122-1132.

26 Stewart EA, McKusick KB, Aggarwal A, Bajorek E, Brady S, Chu A et al. An STSbased radiation hybrid map of the human genome. Genome Res 1997; 7: 422-433.

27 Greenfield EA. Antibodies: A Laboratory Manual. 2nd edn Cold Spring Harbor Laboratory Press: Cold Spring Harbor, NY, USA, 2013.

28 Bloom JS, Ehrenreich IM, Loo WT, Lite TLV, Kruglyak L. Finding the sources of missing heritability in a yeast cross. Nature 2013; 494: 234-237.

29 Doerge RW, Churchill GA. Permutation tests for multiple loci affecting a quantitative character. Genetics 1996; 142: 285-294.

30 R Core Team R: A Language and Environment for Statistical Computing. R Foundation for Statistical Computing: Vienna, Austria, 2015.

31 Lynch M, Walsh B. Genetics and Analysis of Quantitative Traits. Sinauer Associates: Sunderland, MA, USA, 1998.

32 Kahm M, Hasenbrink G, Lichtenberg-Fraté H, Ludwig J, Kschischo M. grofit: fitting biological growth curves with R. J Stat Software 2010; 33: 1-21.

33 Hao HX, Xie Y, Zhang Y, Charlat O, Oster E, Avello M et al. ZNRF3 promotes Wnt receptor turnover in an R-spondin-sensitive manner. Nature 2012; 485: 195-200.

34 Royston P. Remark AS R94: a remark on algorithm AS 181: the W test for normality. Appl Stat 1995; 44: 547-551.

35 Fox J, Weisberg S. An R Companion to Applied Regression. 2nd (edn). Sage: Thousand Oaks, CA, USA, 2011.

36 Champely S. pwr: Basic Functions for Power Analysis 2015. R package version 1.1-2.

37 Visscher PM, Brown MA, McCarthy MI, Yang J. Five years of GWAS discovery. Am J Hum Genet 2012; 90: 7-24.

38 Brunton L, Chabner B, Knollman B. Goodman and Gilman's The Pharmacological Basis of Therapeutics. McGraw-Hill Professional: New York, NY, USA, 2011.

39 Marks PA, Breslow R. Dimethyl sulfoxide to vorinostat: development of this histone deacetylase inhibitor as an anticancer drug. Nat Biotechnol 2007; 25: 84-90.

40 Lewinger JP, Lee SSF, Biernacka J, Wu LY, Shi HS, Bull SB. Comparison of familybased association tests in chromosome regions selected by linkage-based confidence intervals. BMC Genet 2005; 6 (Suppl 1): S62.

41 Koo BK, Spit M, Jordens I, Low TY, Stange DE, van de Wetering $M$ et al. Tumour suppressor RNF43 is a stem-cell E3 ligase that induces endocytosis of Wnt receptors. Nature 2012; 488: 665-669.

42 Xu S. Theoretical basis of the Beavis effect. Genetics 2003; 165: 2259-2268.

43 Glessner JT, Wang K, Cai G, Korvatska O, Kim CE, Wood S et al. Autism genomewide copy number variation reveals ubiquitin and neuronal genes. Nature 2009; 459: $569-573$.

44 Zuko A, Kleijer KTE, Oguro-Ando A, Kas MJH, van Daalen E, van der Zwaag B et al. Contactins in the neurobiology of autism. Eur J Pharmacol 2013; 719: 63-74.

45 Bertino JR. Cancer research: from folate antagonism to molecular targets. Best Pract Res Clin Haematol 2009; 22: 577-582.

46 Schimke RT. Gene amplification; what are we learning? Mutat Res 1992; 276: 145-149.

47 Watson JM, McKay LM, Marshall Graves JA. Genome instability in interspecific cell hybrids II. Aminopterin resistance and gene amplification in lines arising from fusions of cells from divergent mammalian species. J Genet 1988; 67: 75-86.

48 Proffitt KD, Madan B, Ke Z, Pendharkar V, Ding L, Lee MA et al. Pharmacological inhibition of the Wnt acyltransferase PORCN prevents growth of WNT-driven mammary cancer. Cancer Res 2013; 73: 502-507.

49 Hastie SB. Interactions of colchicine with tubulin. Pharmacol Ther 1991; 51: 377-401.

50 Henningsson A, Karlsson MO, Viganò L, Gianni L, Verweij J, Sparreboom A. Mechanism-based pharmacokinetic model for paclitaxel. J Clin Oncol 2001; 19: 4065-4073.

51 Jordan MA, Wilson L. Microtubules as a target for anticancer drugs. Nat Rev Cancer 2004; 4: 253-265.

52 Niel E, Scherrmann JM. Colchicine today. Joint Bone Spine 2006; 73: 672-678.

53 Parness J, Horwitz SB. Taxol binds to polymerized tubulin in vitro. J Cell Biol 1981; 91: 479-487.

54 Ross JL, Santangelo CD, Makrides V, Fygenson DK. Tau induces cooperative Taxol binding to microtubules. Proc Natl Acad Sci USA 2004; 101: 12910-12915.
55 Swanton C, Marani M, Pardo O, Warne PH, Kelly G, Sahai E et al. Regulators of mitotic arrest and ceramide metabolism are determinants of sensitivity to paclitaxel and other chemotherapeutic drugs. Cancer Cell 2007; 11: 498-512.

56 Finkelstein $Y$, Aks SE, Hutson JR, Juurlink DN, Nguyen P, Dubnov-Raz G et al. Colchicine poisoning: the dark side of an ancient drug. Clin Toxicol 2010; 48: 407-414.

57 Michalakis J, Georgatos SD, Romanos J, Koutala H, Georgoulias V, Tsiftsis D et al. Micromolar taxol, with or without hyperthermia, induces mitotic catastrophe and cell necrosis in HeLa cells. Cancer Chemother Pharmacol 2005; 56: 615-622.

58 Dumontet C, Jordan MA. Microtubule-binding agents: a dynamic field of cancer therapeutics. Nat Rev Drug Discov 2010; 9: 790-803.

59 Assié G, Letouz'e E, Fassnacht M, Jouinot A, Luscap W, Barreau O et al. Integrated genomic characterization of adrenocortical carcinoma. Nat Genet 2014; 46: 607-612.

60 de Lau W, Peng WC, Gros P, Clevers H. The R-spondin/Lgr5/Rnf43 module: regulator of Wnt signal strength. Genes Dev 2014; 28: 305-316.

61 Juhlin CC, Goh G, Healy JM, Fonseca AL, Scholl UI, Stenman A et al. Whole-exome sequencing characterizes the landscape of somatic mutations and copy number alterations in adrenocortical carcinoma. J Clin Endocrinol Metab 2015; 100: E493-E502.

62 Anastas JN, Moon RT. Wnt signalling pathways as therapeutic targets in cancer. Nat Rev Cancer 2013; 13: 11-26.

63 Baarsma HA, Königshoff M, Gosens R. The WNT signaling pathway from ligand secretion to gene transcription: molecular mechanisms and pharmacological targets. Pharmacol Ther 2013; 138: 66-83.

64 Rao TP, Kühl M. An updated overview on Wnt signaling pathways: a prelude for more. Circ Res 2010; 106: 1798-1806.

65 Stolz A, Neufeld K, Ertych N, Bastians H. Wnt-mediated protein stabilization ensures proper mitotic microtubule assembly and chromosome segregation. EMBO Rep 2015; 16: 490-499.

66 Stamos JL, Weis WI. The $\beta$-catenin destruction complex. Cold Spring Harb Perspect Biol 2013; 5: a007898.

67 Chikazawa N, Tanaka H, Tasaka T, Nakamura M, Tanaka M, Onishi H et al. Inhibition of Wnt signaling pathway decreases chemotherapy-resistant side-population colon cancer cells. Anticancer Res 2010; 30: 2041-2048.

68 Ciani L, Krylova O, Smalley MJ, Dale TC, Salinas PC. A divergent canonical WNTsignaling pathway regulates microtubule dynamics: dishevelled signals locally to stabilize microtubules. J Cell Biol 2004; 164: 243-253.

69 Gundersen GG, Cook TA. Microtubules and signal transduction. Curr Opin Cell Biol 1999; 11: 81-94.

70 Ling $Y$, Zhong Y, Perez-Soler R. Disruption of cell adhesion and caspase-mediated proteolysis of $\beta$ - and $\gamma$-catenins and APC protein in paclitaxel-induced apoptosis. Mol Pharmacol 2001; 59: 593-603.

71 Ajima R, Hamada H. Wnt signalling escapes to cilia. Nat Cell Biol 2011; 13: 636-637.

$72 \mathrm{He}$ X. Cilia put a brake on Wnt signalling. Nat Cell Biol 2008; 10: 11-13.

73 Huang P, Schier AF. Dampened Hedgehog signaling but normal Wnt signaling in zebrafish without cilia. Development 2009; 136: 3089-3098.

74 May-Simera HL, Kelley MW. Cilia, Wnt signaling, and the cytoskeleton. Cilia 2012; 1: 7.

75 Wallingford JB, Mitchell B. Strange as it may seem: the many links between Wnt signaling, planar cell polarity, and cilia. Genes Dev 2011; 25: 201-213.

76 Kahn M. Can we safely target the WNT pathway? Nat Rev Drug Discov 2014; 13 : 513-532.

77 Kashiwabara K, Yamane H, Tanaka H. Toxicity and prognosis in overweight and obese women with lung cancer receiving carboplatin-paclitaxel doublet chemotherapy. Cancer Invest 2013; 31: 251-257.

78 Jayaprakash V, Ansell G, Galler D. Colchicine overdose: the devil is in the detail. NZ Med J 2007; 120: U2402.

79 Ehrenreich IM, Torabi N, Jia Y, Kent J, Martis S, Shapiro JA et al. Dissection of genetically complex traits with extremely large pools of yeast segregants. Nature 2010; 464: 1039-1042.

80 Michelmore RW, Paran I, Kesseli RV. Identification of markers linked to diseaseresistance genes by bulked segregant analysis: a rapid method to detect markers in specific genomic regions by using segregating populations. Proc Natl Acad Sci USA 1991; 88: 9828-9832.

(2) This work is licensed under a Creative Commons Attributioncc. Non ${ }_{\text {BA }}$ Nommercial-ShareAlike 4.0 International License. The images or other third party material in this article are included in the article's Creative Commons license, unless indicated otherwise in the credit line; if the material is not included under the Creative Commons license, users will need to obtain permission from the license holder to reproduce the material. To view a copy of this license, visit http:// creativecommons.org/licenses/by-nc-sa/4.0/

Supplementary Information accompanies the paper on the The Pharmacogenomics Journal website (http://www.nature.com/tpj) 